\title{
Longitudinal Image Registration with Non-uniform Appearance Change
}

\author{
Istvan Csapo ${ }^{1}$, Brad Davis ${ }^{3}$, Yundi Shi ${ }^{1}$, Mar Sanchez $^{4}$, Martin Styner ${ }^{1}$, \\ and Marc Niethammer ${ }^{1,2}$ \\ 1 University of North Carolina at Chapel Hill, NC \\ 2 Biomedical Research Imaging Center, UNC Chapel Hill, NC \\ ${ }^{3}$ Kitware, Inc., Carrboro, NC \\ 4 Emory University, Atlanta, GA \\ icsapo@cs.unc.edu
}

\begin{abstract}
Longitudinal imaging studies are frequently used to investigate temporal changes in brain morphology. Image intensity may also change over time, for example when studying brain maturation. However, such intensity changes are not accounted for in image similarity measures for standard image registration methods. Hence, (i) local similarity measures, (ii) methods estimating intensity transformations between images, and (iii) metamorphosis approaches have been developed to either achieve robustness with respect to intensity changes or to simultaneously capture spatial and intensity changes. For these methods, longitudinal intensity changes are not explicitly modeled and images are treated as independent static samples. Here, we propose a model-based image similarity measure for longitudinal image registration in the presence of spatially non-uniform intensity change.
\end{abstract}

\section{Introduction}

To study changes that occur during brain development, neurodegeneration, or disease progression in general, longitudinal imaging studies are important. Spatial correspondences almost always need to be established between images for longitudinal analysis through image registration. Most image registration methods have been developed to

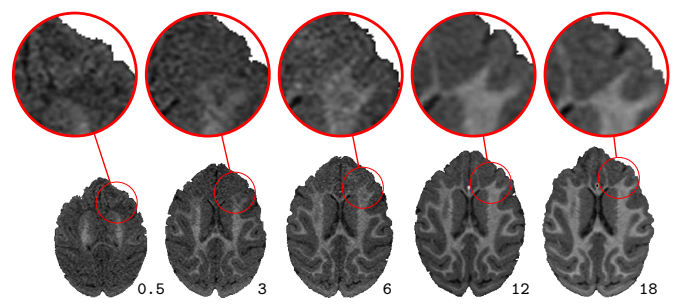

Fig. 1. Brain slices and magnifications for a monkey at ages 2 weeks, $3,6,12$, and 18 months. White matter appearance changes locally as axons are myelinated during brain development. align images that are similar in appearance or structure. If such similarity is not given (e.g., in case of pathologies or for pre- and post-surgery images) cost function masking is typically used to discard image regions without correspondence from the registration. Such strict exclusion is not always desirable. When investigating brain maturation for example (our target application in this paper) valid correspondences for the complete brain are expected to exist. However, 
brain appearance changes continuously over time due to biological tissue changes (here, myelination of white matter [112]) and adversely affects image registration results 9 .

The effect of appearance change on the result of an image registration depends on the chosen transformation model and the chosen image similarity measure. Generally, transformation models with few degrees of freedom (such as rigid or affine transformations) are affected less by local changes in image appearance than transformation models which can capture localized spatial changes, such as elastic or fluid models. In particular, we have previously shown that affine methods perform well even in the presence of strong non-uniform appearance change, while deformable methods introduce erroneous local deformations in order to resolve inconsistencies in appearance [3. However, transformation models which can capture local deformations are desirable for many longitudinal studies as changes in morphology tends to be spatially non-uniform.

For longitudinal registration, temporal regularization of the transformation model has been explored recently. This is motivated by the assumption that unrealistic local changes can be avoided by enforcing temporal smoothness of a transformation [5/7]. In this paper we instead focus on the complementary problem of determining an appropriate image similarity measure for longitudinal registration in the presence of temporal changes in image intensity.

Approaches which address non-uniform intensity changes have mainly addressed registration for image-pairs so far and either rely on local image uniformities 913 or try to estimate image appearance changes jointly with an image transform 81110]. Often (e.g., for bias field compensation in magnetic resonance imaging), image intensity changes are assumed to be smooth. Our proposed approach in contrast, estimates local longitudinal models of intensity change using all available images. Our approach alternates between parameter estimation for the local models of intensity change and estimation of the spatial transformation. Image similarities are computed relative to the estimated intensity models, hence accounting for local changes in image intensities.

Section 2 introduces the model-based image similarity measure (mSM). Section 3 discusses parameter estimation. Section 4 describes the performed experiments and discusses results. The paper concludes with a summary and outlook on future work.

\section{Model-Based Similarity Measure}

Assume we have an image intensity model $\hat{I}(x, t ; p)$ which for a parameterization, $p$, describes the expected intensity values for a given point $x$ at a time $t$. This model is defined in a spatially fixed target image. Then, instead of registering a measured image $I_{i}$ at $t_{i}$ to a fixed target image $I_{T}$ we can register it to its corresponding intensity-adjusted image $\hat{I}\left(x, t_{i} ; p\right)$, effectively removing temporal intensity changes for a good model and a good parameterization, $p$. Hence,

$$
\operatorname{Sim}\left(I_{i} \circ \Phi_{i}, I_{T}\right) \text { is replaced by } \operatorname{Sim}\left(I_{i} \circ \Phi_{i}, \hat{I}\left(x, t_{i} ; p\right)\right),
$$


where $\operatorname{Sim}(\cdot, \cdot)$ is any chosen similarity measure (e.g., sum of squared differences (SSD), normalized cross correlation, or mutual information), and $\Phi_{i}$ is the map from image $I_{i}$ to the spatially fixed target space. Since our method aims to create an intensity adjusted model $\hat{I}$ that matches the appearance of the source image, we use SSD in this paper. We call the intensity-adjusted SSD similarity measure a sum of squared residual (SSR) model, where the residual is defined as the difference between the predicted and the measured intensity value.

\subsection{General Local Intensity Model Estimation for SSD}

Since SSR is a local image similarity measure, for a given set of $N$ measurement images $\left\{I_{i}\right\}$ at times $\left\{t_{i}\right\}$ we can write the full longitudinal similarity measure as the sum over the individual SSRs, i.e.,

$$
S S R\left(\left\{I_{i}\right\} ; p\right)=\sum_{i=0}^{N-1} \int_{\Omega}\left(I_{i} \circ \Phi_{i}(x)-\hat{I}\left(x, t_{i} ; p\right)\right)^{2} d x,
$$

where $\Omega$ is the image domain of the fixed image. For given spatial transforms $\Phi_{i}$ this is simply a least-squares parameter estimation problem given the measurements $\left\{I_{i} \circ \Phi_{i}(x)\right\}$ and the predicted model values $\left\{\hat{I}\left(x, t_{i} ; p\right)\right\}$. We use alternating optimization with respect to the intensity model parameters, $p$, and the spatial transformations $\Phi_{i}$ to convergence (see Sec. 33).

\subsection{Logistic Intensity Model with Elastic Deformation}

SSR can be combined with any model for intensity change, ranging from a given constant target image (the trivial model), linear models, and splines to models more closely adapted to the myelination process we are interested in capturing during neurodevelopment. Since the myelination process exhibits a rapid increase during early brain development followed by a gradual leveling off [4], nonlinear appear-

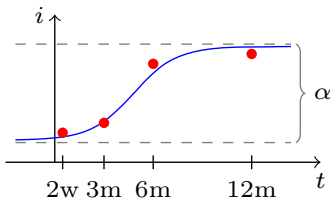

Fig. 2. Logistic intensity model ance models are justified. In this paper we investigate the logistic model

$$
\hat{I}(x, t ; \alpha(x), \beta(x), k(x))=\frac{\alpha(x)}{1+\beta(x) e^{-k(x) t}},
$$

which is often used in growth studies [6. Here, $\alpha, \beta$, and $k$ are spatially varying model parameters with biological meaning, $k$ being the maximum rate of intensity change, $\alpha$ the maximum increase of white matter intensity during myelination, and $\beta$ is related to the onset time of myelination (see Fig. 2). Assuming that both unmyelinated and fully myelinated white matter intensities are spatially uniform we keep $\alpha$ constant as the difference between myelinated (upper asymptote) and unmyelinated (lower asymptote) white matter intensities. This is a simplifying, but reasonable assumption since intensity inhomogeneities in 
unmyelinated or myelinated white matter are small compared to the white matter intensity change due to the myelination process itself [1].

\section{Parameter Estimation}

Once the parameters for the local intensity models are known, SSR can be used to replace the image similarity measure in any longitudinal registration method. Here, we use an elastic deformation model (Sec. 3.1) and jointly estimate the parameters for the intensity model (Sec. 3.2).

\subsection{Registration Model}

The growth process of the brain not only includes appearance change but complex morphological changes as well, hence the need for a deformable transformation model. To single out plausible deformations, we use (for simplicity) an elastic regularizer 2] defined on the displacement field $u$ as

$$
S[u]=\int_{\Omega} \frac{\mu}{4} \sum_{j, k=1}^{d} \underbrace{\left(\partial_{x_{j}} u_{k}+\partial_{x_{k}} u_{j}\right)^{2}}_{\text {rigidity }}+\frac{\lambda}{2} \underbrace{(\operatorname{div} u)^{2}}_{\text {volume change }} d x
$$

where $\mu(=1)$ and $\lambda(=0)$ are the Lamé constants that control elastic behavior, and the div is the divergence operator defined as $\nabla \cdot u$, where $\nabla$ is the gradient operator. Registrations over time then decouple into pairwise registration between the intensity-adjusted target image and a given source image $I_{i}$. This is sufficient for our test of the longitudinal image similarity measure, but could easily be combined with a spatio-temporal regularizer which would then directly couple the transformations between the images of a time-series (instead of only having an indirect coupling through the model-based similarity measure).

\subsection{Model Parameter Estimation}

We estimate the intensity model parameters only within the white matter (segmentation was obtained at the last time-point with an atlas-based segmentation method and propagated to earlier time-points [14]) where image appearance changes non-uniformly over time; for simplicity, gray matter intensity was assumed to stay constant.

Note that overestimating the white matter results in fitting the model to the surrounding gray matter voxels. This has negligible effect on the registration, since the model can capture the constant gray matter intensities. Underestimating the white matter, on the other hand, can lead to uncorrected intensities near the white-gray matter boundary and introduce erroneous local deformations.

Instead of estimating the parameters independently for each voxel, spatial regularization was achieved by estimating the parameters from overlapping local $3 \times 3 \times 3$ neighborhoods using robust statistics (median of the parameters). 
The algorithm is defined as follows

0) Initialize model $\hat{I}$ parameters to $p=p_{0}$.

1) Affinely pre-register images $\left\{I_{i}\right\}$ to $\hat{I}$.

2) Estimate the appearance of $\hat{I}$ at times $\left\{t_{i}\right\}$, giving $\left\{\hat{I}\left(t_{i}\right)\right\}$.

3) Estimate displacement fields $\left\{u_{i}\right\}$ by registering images $\left\{I_{i}\right\}$ to $\left\{\hat{I}\left(t_{i}\right)\right\}$.

4) Estimate model parameters $p$ from the registered images $\left\{I_{i} \circ u_{i}\right\}$.

5) Repeat from step 2 until convergence.

The algorithm terminates once the registration energy decreases by less than a given tolerance between subsequent iterations. In all our experiments only few iterations (typically less than 5) were required. A more in-depth numerical convergence analysis should be part of future work. If desired, a prior model defined in the target image (a form of intensity model parameter atlas) could easily be integrated into this framework.

\section{Experimental Results}

We compared the modelbased similarity measure to mutual information (MI) on sets of longitudinal magnetic resonance

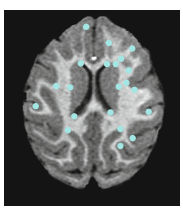

$12 \mathrm{mo}$

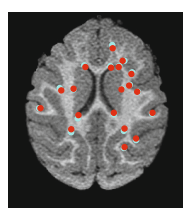

$6 \mathrm{mo}$

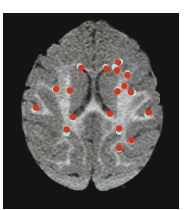

$3 \mathrm{mo}$

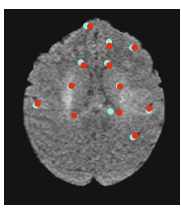

$2 \mathrm{wk}$

Fig. 3. Corresponding target (cyan) and source (red) landmarks for a single subject images of 9 monkeys, each with 4 time-points. Each set was affinely pre-registered and intensity normalized so that the gray matter intensity distributions matched after normalization (gray matter intensity generally stays constant over time).

We registered $3 \mathrm{D}$ images of the three early time-points $I_{2 \mathrm{wk}}, I_{3 \mathrm{mo}}, I_{6 \mathrm{mo}}$ to the target image $I_{12 \mathrm{mo}}$ with an elastic registration method. Since the ground truth deformations were not known, manually selected landmarks (Fig. 3) identified corresponding regions of the brain at the different time-points (10-20 landmarks in a single slice for each of the 4 time-points in all 9 subjects; the landmarks were picked based on geometric considerations). The distance between transformed and target landmarks yielded registration accuracy. Figure 4 shows the experimental setup.

Note that for the model-based method the target image is not $I_{12 \mathrm{mo}}$ but the model $\hat{I}_{12 \mathrm{mo}}$ estimated at time $t$ of the source image. Here, we estimate the intensity change of $I_{12 \mathrm{mo}}$ as white matter segmentation is easily obtained given the good gray matter white matter contrast, but other time-points could be used.

With MI, the registration method accounts for both the non-uniform white matter appearance change and the morphological changes due to growth through large local deformations. This is especially apparent for registrations between $I_{2 \mathrm{wk}}$ (and to a lesser extent $I_{3 \mathrm{mo}}$ ) and the target $I_{12 \mathrm{mo}}$ and suggests large 


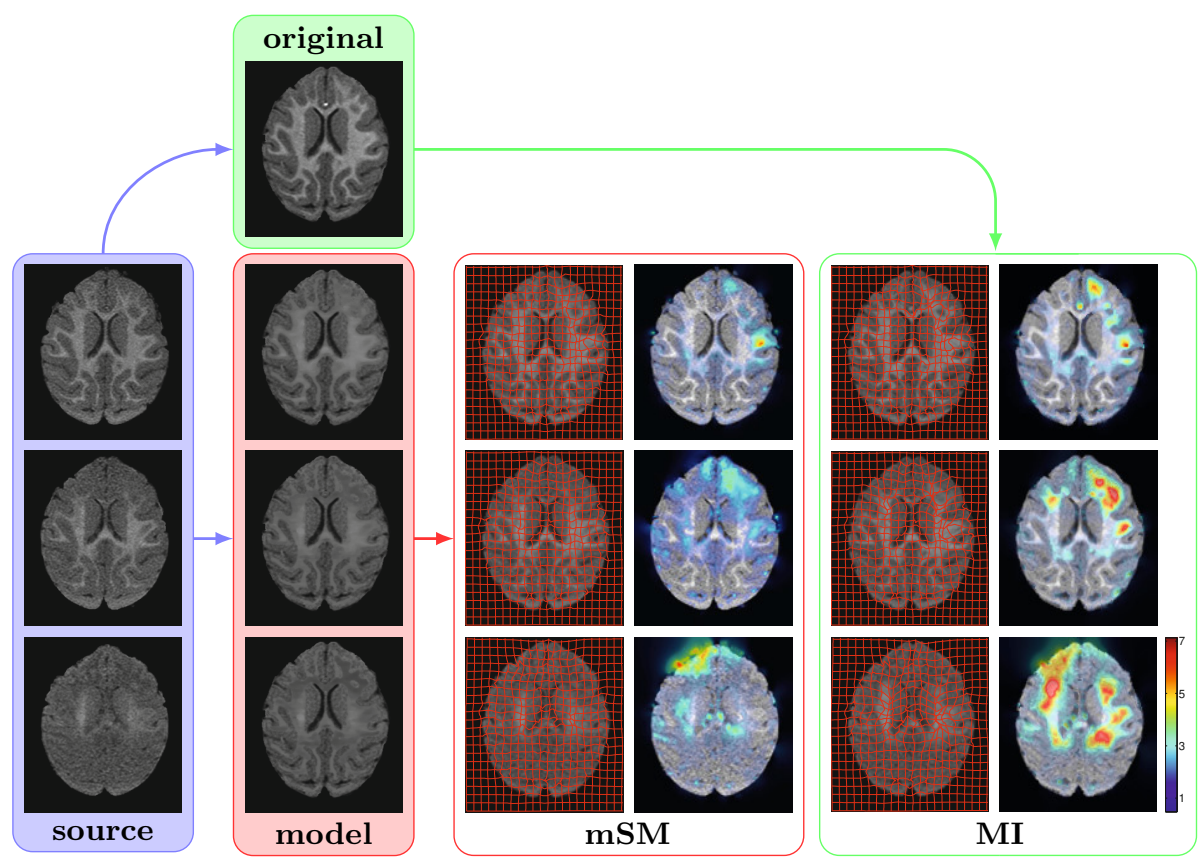

Fig. 4. Experimental setup and results for a single subject. To test MI, the source images (blue; from bottom: $I_{2 \mathrm{wk}}, I_{3 \mathrm{mo}}, I_{6 \mathrm{mo}}$ ) are registered to the latest time-point $I_{12 \mathrm{mo}}$ (green). The resulting deformation field and the magnitude of the deformations (in pixels) is shown in the right panel. For mSM, the source images are registered to the model (red) that estimates the appearance of $I_{12 \mathrm{mo}}$ at the corresponding time of each source image (results in middle panel).

local morphological changes contradictory to normal brain development [4. The landmark mismatch results (Fig. 5) show that both mutual information and the model-based approach perform well in the absence of large intensity nonuniformity, however, mSM consistently introduces smaller erroneous deformations than MI.

Table 1 shows the aggregate results of the landmark mismatch calculations for both methods. The model-based approach can account for appearance change by adjusting the intensity of the model image (see the estimated model images in Fig. 4) and therefore is most beneficial when the change in appearance between the source and target image is large $\left(I_{2 \mathrm{wk}}, I_{3 \mathrm{mo}}\right)$.

We also compared $1^{\text {st }}$ and $2^{\text {nd }}$ degree polynomial intensity models to the logistic model and found no significant difference. This is expected with only 4 time-points as even the $1^{\text {st }}$ degree model can reasonably estimate the local appearance changes. However, we expect the logistic model to outperform the simpler models in larger studies with more time-points or when the model appearance needs to be extrapolated (e.g., if new images are acquired later in a longitudinal study after the previous time-points have been aligned). This will be investigated as part of future work. 


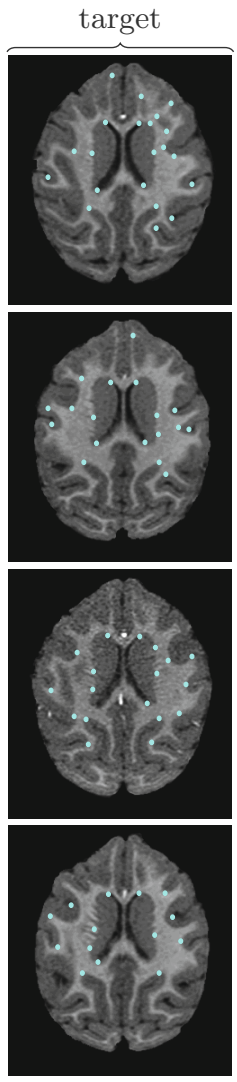

$12 \mathrm{mo}$
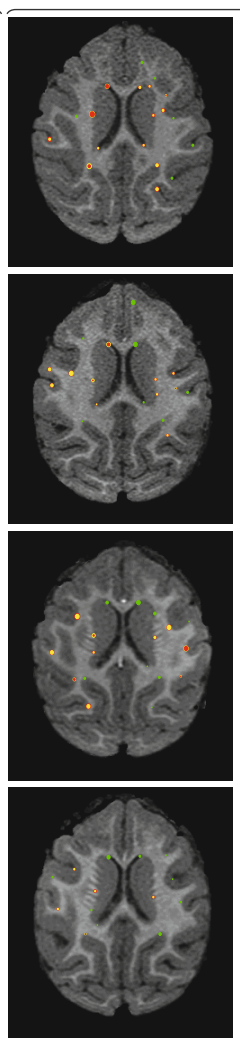

$6 \mathrm{mo}$ source
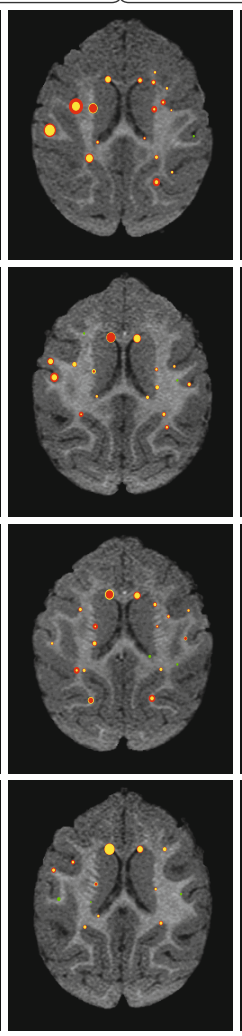

$3 \mathrm{mo}$
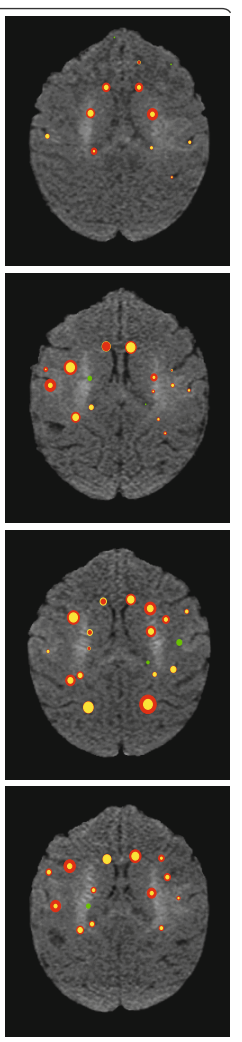

2 wk

Fig. 5. Landmark registration error. Each row shows a single subject. The first column shows the target images and landmarks (cyan). Columns 2-4 are the source images and landmarks: each source landmark is marked as two circles (red: MI, yellow: mSM) with size proportional to registration error (smaller circle on top; green: both are equal). That is, the size of the circles is proportional to registration accuracy (smaller is more accurate) in that particular location.

Table 1. Landmark registration error (in voxels) between target $I_{12 \mathrm{mo}}$ and source images $I_{2 \mathrm{wk}}, I_{3 \mathrm{mo}}, I_{6 \mathrm{mo}}$ (significance level is $\alpha=0.05$; significant results are highlighted).

\begin{tabular}{|c|c|c|c|c|c|}
\hline \multicolumn{3}{|r|}{$I_{2 \mathrm{wk}}$} & \multicolumn{2}{|r|}{$I_{3 \mathrm{mo}}$} & $I_{6 \mathrm{mo}}$ \\
\hline & mean & std $50^{\text {th }} 90^{\text {th }}$ & mean & std $50^{\text {th }} 90^{\text {th }}$ & mean std $50^{\text {th }} 90^{\text {th }}$ \\
\hline MI & 1.76 & $\begin{array}{lll}1.09 & 1.60 & 3.22\end{array}$ & 1.07 & $\begin{array}{lll}0.77 & 0.84 & 2.04\end{array}$ & $\begin{array}{llll}0.66 & 0.46 & 0.56 & 1.22\end{array}$ \\
\hline $\mathrm{mSM}$ & 1.15 & 0.840 .932 .24 & 0.74 & 0.570 .541 .66 & $\begin{array}{llll}0.61 & 0.39 & 0.50 & 1.18\end{array}$ \\
\hline
\end{tabular}




\section{Conclusions}

We proposed a new model-based similarity measure which allows the deformable registration of longitudinal images with appearance change. This method can account for the intensity change over time and enables the registration method to recover the deformation due only to changes in morphology. We compared the model-based approach to mutual information and demonstrated that it can achieve higher accuracy than mutual information in cases when there is a large appearance change between source and target images. We used a logistic model of intensity change and an elastic deformation model, however, the formulation is general and can be used with any other appearance or deformation model. In the future we will investigate the use of prior models to inform the estimation step in regions with high uncertainty (e.g., due to poor initial alignment), in addition to the effect of various intensity models on the registration accuracy.

Acknowledgments. This work was supported by NSF EECS-1148870, NSF EECS-0925875, NIH NIHM 5R01MH091645-02, NIH NIBIB 5P41EB002025-28, U54 EB005149, P50 MH078105-01A2S1, P50 MH078105-01.

\section{References}

1. Barkovich, A.J., Kjos, B.O., Jackson, D.E., Norman, D.: Normal maturation of the neonatal and infant brain: MR imaging at 1.5T. Radiology 166, 173-180 (1988)

2. Broit, C.: Optimal registration of deformed images. Ph.D. thesis, University of Pennsylvania (1981)

3. Csapo, I., Davis, B., Shi, Y., Sanchez, M., Styner, M., Niethammer, M.: Temporally-Dependent Image Similarity Measure for Longitudinal Analysis. In: Dawant, B.M., Christensen, G.E., Fitzpatrick, J.M., Rueckert, D. (eds.) WBIR 2012. LNCS, vol. 7359, pp. 99-109. Springer, Heidelberg (2012)

4. Dobbing, J., Sands, J.: Quantitative growth and development of human brain. Archives of Disease in Childhood 48(10), 757-767 (1973)

5. Durrleman, S., Pennec, X., Trouvé, A., Gerig, G., Ayache, N.: Spatiotemporal Atlas Estimation for Developmental Delay Detection in Longitudinal Datasets. In: Yang, G.-Z., Hawkes, D., Rueckert, D., Noble, A., Taylor, C. (eds.) MICCAI 2009, Part I. LNCS, vol. 5761, pp. 297-304. Springer, Heidelberg (2009)

6. Fekedulegn, D., Siurtain, M.P.M., Colbert, J.J.: Parameter estimation of nonlinear growth models in forestry. Silva Fennica 33(4), 327-336 (1999)

7. Fishbaugh, J., Durrleman, S., Gerig, G.: Estimation of Smooth Growth Trajectories with Controlled Acceleration from Time Series Shape Data. In: Fichtinger, G., Martel, A., Peters, T. (eds.) MICCAI 2011, Part II. LNCS, vol. 6892, pp. 401-408. Springer, Heidelberg (2011)

8. Friston, K., Ashburner, J., Frith, C., Poline, J., Heather, J.D., Frackowiak, R.: Spatial registration and normalization of images. Human Brain Mapping 2, 165-189 (1995)

9. Loeckx, D., Slagmolen, P., Maes, F., Vandermeulen, D., Suetens, P.: Nonrigid image registration using conditional mutual information. IEEE Transactions on Medical Imaging 29(1), 19-29 (2010) 
10. Miller, M.I., Younes, L.: Group actions, homeomorphisms, and matching: A general framework. International Journal of Computer Vision 41(1/2), 61-84 (2001)

11. Roche, A., Guimond, A., Ayache, N., Meunier, J.: Multimodal Elastic Matching of Brain Images. In: Vernon, D. (ed.) ECCV 2000. LNCS, vol. 1843, pp. 511-527. Springer, Heidelberg (2000)

12. Sampaio, R.C., Truwit, C.L.: Myelination in the developing brain. In: Handbook of Developmental Cognitive Neuroscience, pp. 35-44. MIT Press (2001)

13. Studholme, C., Drapaca, C., Iordanova, B., Cardenas, V.: Deformation-based mapping of volume change from serial brain mri in the presence of local tissue contrast change. IEEE Transactions on Medical Imaging 25(5), 626-639 (2006)

14. Styner, M., Knickmeyer, R., Coe, C., Short, S.J., Gilmore, J.: Automatic regional analysis of dti properties in the developmental macaque brain. Society of PhotoOptical Instrumentation Engineers (SPIE) Conference Series, vol. 6914 (2008) 